\title{
Generalized Analysis on Diversity-Multiplexing Trade-off of Relay Protocols
}

\author{
Yafeng Wang ${ }^{1}$, Lin Qin $^{1}$ and Wei Xiang ${ }^{2}$ \\ ${ }^{1}$ Key Laboratory of Universal Wireless Communications, Ministry of Education, Beijing University of Posts and Telecommunications, \\ Beijing, 100876, China \\ ${ }^{2}$ Faculty of Engineering and Surveying, University of Southern Queensland, Toowoomba, QLD 4350, Australian
}

Received: 8 Oct. 2012, Revised: 25 Dec. 2012, Accepted: 28 Dec. 2012

Published online: 1 Jun. 2013

\begin{abstract}
Under the more practical assumption that transmitters have no knowledge of the channel state information (CSI) but the channel distribution information (CDI), this paper examines the diversity-multiplexing trade-off (DMT) of two classes of cooperative protocols, namely, the amplify-and-forward (AF) and decode-and-forward (DF) protocols, with a three-node half-duplex relay network under slow fading. First, analyzing these protocols reveals that each one has its own optimal DMT with a specific time slot ratio, i.e., the ratio between the durations of the slots assigned to base station transmission and that assigned to relay transmission. We then demonstrate that under ideal coding, the incremental decode-and-forward protocol approaches the DMT upper bound when the two slots are of equal length, which outperforms all the other protocols. Furthermore, it is proved that the selective decode-and-forward protocol achieves its optimal performance with a slot assignment ratio of $(\sqrt{5}+1) / 2$. In addition, we also find $\mathrm{AF}$, selective $\mathrm{AF}$ and incremental AF have no difference from the perspective of DMT.
\end{abstract}

Keywords: Cooperative diversity, diversity-multiplexing trade-off, selective relaying, incremental relaying, half duplex

\section{Introduction}

The diversity-multiplexing trade-off (DMT), a metric to evaluate the diversity and degree of freedom gains in general fading channels, was introduced by Zheng and Tse [1] and first applied to wireless relay networks in [2]. The DMT is upper-bounded [2] by that of $2 \times 1$ multiple-input single-output (MISO) channel: $d(r)=2(r-1)$, where $r$ is the multiplexing gain and $d$ is the diversity gain. On the other hand, there have been numerous recent works focusing on DMT, in which many cooperative protocols were proposed to improve diversity gains. These protocols fall into three main categories. The first class is based on amplify-and-forward (AF), such as non-orthogonal amplify-and-forward $[3,4]$ and slotted amplify-and-forward [5,6]. The second category is several improved versions of the decode-and-forward (DF) protocols, such as dynamic decode-and-forward [4]. The third one includes some other protocols, for instance, compress-and-forward $[7,8,9]$. However, none of these protocols are able to achieve the exact bound for the entire range of multiplexing gains when the transmitter has no knowledge of the channel state information (CSI).

Hence, an interesting question arises: under the assumption that the transmitter is aware of the channel distribution information (CDI) instead of the CSI, is it possible for a half-duplex cooperative relay protocol in slow fading to achieve the MISO upper bound? In [2], the incremental amplify-and-forward protocol proposed by Laneman and Tse seems to be able to achieve the bound. This conclusion is, however, obtained subject to the condition that during a unit slot, the total number of correct bits is $R / 2$, and when it is desired to transmit $R$ bit correctly, the protocol is no longer optimal.

Therefore, our work is to investigate and identify a protocol that answers the question raised above.

Our work, though based on [2], still is of substantial difference: 1) In [2], Laneman and Tse focused on repetition coding which would lead to the loss of capacity, whereas we adopt ideal coding to overcome this drawback; 2) For the sake of simplicity, [2] fixed each time slot to an equal length, while in this paper we will demonstrate that the slot length ratio is an essential factor

\footnotetext{
* Corresponding author e-mail: wangyf@bupt.edu.cn
} 
affecting the DMT performance. We will derive the closed-form DMTs of selective decode-and-forward (SDF), incremental decode-and-forward (IDF), amplify-and-forward (AF), selective amplify-and-forward (SAF) and incremental amplify-and-forward (IAF). We demonstrate that the closed-form DMT of IDF is identical to that of the MISO bound for any $0 \leq r \leq 1$. More interestingly, we find all of the DF protocols have their own optimal DMT with a specific slot duration ratio, while AF protocols exhibit no difference in the sense of DMT.

The remainder of this paper is organized as follows. Section II presents the system model used throughout the paper. The DMT analyses for IDF and SDF are detailed in Section III. Section IV is dedicated to the derivation of the DMT of AF protocols. Section V presents numerical results. Finally, Section VI draws the conclusion.

\section{System Model}

\subsection{Relay Protocol}

The system model is illustrated in Figure 1. The network has one relay node and a direct link between the source (S) and destination (D). The lengths of two slots are adjustable depending on the constant $\tau$.

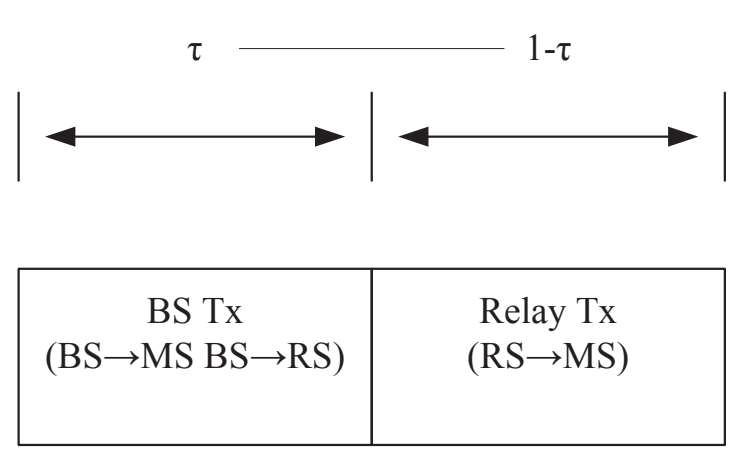

Fig. 1 A fixed half-duplex relaying protocol, where the BS transmits for $\tau$ fraction of time and relay transmits for the remaining $(1-\tau)$ time.

Our work is based on the following conditions:

$-\mathrm{S}$ transmits at a fixed rate of $R$ bps, which means $\mathrm{S}$ should transmit $R$ bits correctly during the unit slot. If not, outage occurs.

-All the channels are assumed to be flat Rayleigh fading channels and the channel gains $h_{s d}, h_{r d}$ and $h_{s r}$ are independent Rayleigh random variables with variances $\sigma_{s d}^{2}, \sigma_{r d}^{2}$ and $\sigma_{s r}^{2}$, respectively. As a result, $\left|h_{s d}\right|^{2},\left|h_{r d}\right|^{2}$ and $\left|h_{s r}\right|^{2}$ are independent exponential random variables with means $1 / \sigma_{s d}^{2}, 1 / \sigma_{r d}^{2}$ and $1 / \sigma_{s r}^{2}$, respectively.

-For each time slot, neither $S$ nor the relay has the knowledge of the CSI, i.e., $h_{s d}, h_{r d}$ and $h_{s r}$. But $\mathrm{S}$ is aware of the distributions of $h_{s d}, h_{r d}$ and $h_{s r}$, namely, the CDI.

-An average transmit power constraint of $P$ is assumed at both the source and the relay. Define $S N R=P / N_{0}$.

\subsection{Definition of DMT}

Let $R=r \log S N R$, then we have [10]

$$
\lim _{S N R \rightarrow \infty} \frac{\log P_{\text {out }}(r \log S N R)}{\log S N R}=-d(r)
$$

where $P_{\text {out }}(R)$ denotes the outage probability at target rate $R$. Equation (1) is one of the possible ways to define the DMT, i.e., the diversity gain $d(r)$ when the multiplexing gain is $r$.

\section{DMT of DF Protocols}

\subsection{Incremental Decode-and-Forward Protocol}

\subsubsection{Protocol Description}

$\mathrm{S}$ transmits its information to $\mathrm{D}$ with spectral efficiency $R$. Then $\mathrm{D}$ indicates the success or failure of the transmission by broadcasting a single bit to feed back to $\mathrm{S}$ and the relay. If successful, the relay keeps silent and $S$ continues to transmit new information to D. Otherwise, the relay will decode and forward what it received from $\mathrm{S}$ by applying ideal coding and S does nothing.

\subsubsection{DMT Performance}

To guarantee that $R$ bits can be successfully transmitted in a unit slot, the maximum average mutual information [11] can be readily shown as

$$
I_{I D F}=\left\{\begin{array}{c}
\tau \log \left(1+\left|h_{s d}\right|^{2} S N R\right), \\
\text { when } \tau \log \left(1+\left|h_{s d}\right|^{2} S N R\right) \geq R \\
\tau \log \left(1+\left|h_{s d}\right|^{2} S N R\right)+(1-\tau) \log \left(1+\left|h_{r d}\right|^{2} S N R\right), \\
\text { when } \tau \log \left(1+\left|h_{s d}\right|^{2} S N R\right)<R
\end{array}\right.
$$


The outage probability for IDF can be computed according to

$$
\begin{aligned}
& P_{\text {out }}^{I D F}=\operatorname{Pr}\left[\tau \log \left(1+\left|h_{\text {sd }}\right|^{2} S N R\right)<R\right] . \\
& \operatorname{Pr}\left[\tau \log \left(1+\left|h_{s d}\right|^{2} S N R\right)+(1-\tau) \log \left(1+\left|h_{r d}\right|^{2} S N R\right)\right. \\
& \left.<R \mid \tau \log \left(1+\left|h_{s d}\right|^{2} S N R\right)<R\right] \\
& =\operatorname{Pr}\left[\tau \log \left(1+\left|h_{s d}\right|^{2} S N R\right)+(1-\tau) \log \left(1+\left|h_{r d}\right|^{2} S N R\right)<R\right]
\end{aligned}
$$

Let $u=\left|h_{s d}\right|^{2}$ and $v=\left|h_{r d}\right|^{2}$, then (3) becomes

$$
\operatorname{Pr}[\tau \log (1+u S N R)+(1-\tau) \log (1+v S N R)<R]
$$$$
=\operatorname{Pr}\left[(1+u S N R)^{\tau}(1+v S N R)^{(1-\tau)}<2^{R}\right]
$$$$
=\int_{0}^{+\infty} \operatorname{Pr}\left[(1+u S N R)^{\tau}(1+v S N R)^{(1-\tau)}<2^{R} \mid V=v\right] p_{V}(v) d v
$$$$
=\int_{0}^{g\left(\frac{1}{S N R}\right)} \operatorname{Pr}\left[u<\frac{\left(\frac{2^{R / \tau}}{(1+v S N R)^{\frac{1-\tau}{\tau}}}\right)-1}{S N R} \mid V=v\right] \lambda_{V} e^{-\lambda_{V} v} d v
$$$$
=\int_{0}^{g\left(\frac{1}{S N R}\right)}\left[1-\exp \left\{-\lambda_{U}\left[\frac{\left(\frac{2^{R}}{(1+v S N R)^{1-\tau}}\right)^{\frac{1}{\tau}}-1}{S N R}\right]\right\}\right] \lambda_{V} e^{-\lambda_{V} v} d v
$$

where $g\left(\frac{1}{S N R}\right)=\frac{2 \frac{R}{1-\tau}-1}{S N R}$.

To upper bound (4), we use the inequalities $1-e^{-x} \leq x$ for all $x \geq 0$, and $e^{-y} \leq 1$ for all $y \geq 0$ [2], so that (4) is upper-bounded by

$$
\begin{aligned}
& \int_{0}^{g\left(\frac{1}{S N R}\right)} \lambda_{U}\left[\frac{\left(\frac{2^{R}}{(1+v S N R)^{1-\tau}}\right)^{\frac{1}{\tau}}-1}{S N R}\right] \lambda_{V} d v \\
& =\frac{\lambda_{U} \lambda_{V}}{S N R}\left[\frac{2^{\frac{R}{\tau}}}{S N R} \int_{0}^{g\left(\frac{1}{S N R}\right)}\left(\frac{1}{(1+v S N R)^{1-\tau}}\right)^{\frac{1}{\tau}} \cdot\right. \\
& \left.\quad d(1+v S N R)-g\left(\frac{1}{S N R}\right)\right] \\
& =\frac{\lambda_{U} \lambda_{V}}{S N R}\left\{\frac{2^{\frac{R}{\tau}}}{S N R} \frac{\tau}{2 \tau-1}\left[\left(2^{\left.\frac{R}{1-\tau}\right)^{\frac{2 \tau-1}{\tau}}}-1\right]-g\left(\frac{1}{S N R}\right)\right\}\right.
\end{aligned}
$$

Hence,

$$
\begin{aligned}
\limsup _{S N R \rightarrow \infty} & \operatorname{Pr}\left[\tau \log \left(1+\left|h_{S d}\right|^{2} S N R\right)+\right. \\
& \left.(1-\tau) \log \left(1+\left|h_{r d}\right|^{2} S N R\right)<R\right] \\
& =\frac{\lambda_{U} \lambda_{V}}{S N R^{2}} \frac{\tau}{2 \tau-1}\left[\left(2^{\left.\left.\frac{R}{1-\tau}\right)^{\frac{2 \tau-1}{\tau}}-1\right] 2^{\frac{R}{\tau}}}\right.\right.
\end{aligned}
$$

To lower bound (4), we make use of the concavity of $1-e^{-x}$. That is, for any $t \geq 0$ for all $x \leq t$ [2], the following is true

$$
1-e^{-x} \geq \frac{1-e^{-t}}{t} x
$$

Along with another inequality $e^{-y} \geq 1-y$ for all $y \geq 0$, we can lower bound (4) by

$$
\begin{aligned}
& \int_{0}^{g\left(\frac{1}{S N R}\right)} \frac{1-e^{-t}}{t} \lambda_{U}\left[\frac{\left(\frac{2^{R}}{(1+v S N R)^{1-\tau}}\right)^{\frac{1}{\tau}}-1}{S N R}\right] \lambda_{V}\left(1-\lambda_{V} v\right) d v \\
& \geq \frac{\lambda_{U} \lambda_{V}}{S N R} \frac{1-e^{-t}}{t}\left[1-\lambda_{V} g\left(\frac{1}{S N R}\right)\right] \cdot \\
& \quad \int_{0}^{g\left(\frac{1}{S N R}\right)}\left[\left(\frac{2^{R}}{\left.\left.(1+v S N R)^{1-\tau}\right)^{\frac{1}{\tau}}-1\right] d v}\right.\right. \\
& =\frac{\lambda_{U} \lambda_{V}}{S N R} \frac{1-e^{-t}}{t}\left[1-\lambda_{V} g\left(\frac{1}{S N R}\right)\right] \cdot \\
& \quad\left\{\frac { 2 ^ { \frac { R } { \tau } } } { S N R } \frac { \tau } { 2 \tau - 1 } \left[\left(2^{\left.\left.\left.\frac{R}{1-\tau}\right)^{\frac{2 \tau-1}{\tau}}-1\right]-g\left(\frac{1}{S N R}\right)\right\}}\right.\right.\right.
\end{aligned}
$$

where $t=\lambda_{U} \frac{2^{\frac{R}{\tau}}}{S N R}$.

Hence,

$$
\begin{aligned}
\liminf _{S N R \rightarrow \infty} & \operatorname{Pr}\left[\tau \log \left(1+\left|h_{S d}\right|^{2} S N R\right)+\right. \\
& \left.(1-\tau) \log \left(1+\left|h_{r d}\right|^{2} S N R\right)<R\right] \\
& =\frac{\lambda_{U} \lambda_{V}}{S N R^{2}} \frac{\tau}{2 \tau-1}\left[\left(2^{\frac{R}{1-\tau}}\right)^{\frac{2 \tau-1}{\tau}}-1\right] 2^{\frac{R}{\tau}}
\end{aligned}
$$

Since the bounds in (6) and (9) are equivalent, we arrive at

$$
\begin{aligned}
& \operatorname{Pr}\left[\tau \log \left(1+\left|h_{S d}\right|^{2} S N R\right)+(1-\tau) \log \left(1+\left|h_{r d}\right|^{2} S N R\right)<R\right] \\
& \sim \frac{\lambda_{U} \lambda_{V}}{S N R^{2}} \frac{\tau}{2 \tau-1}\left[2^{\frac{R(2 \tau-1)}{\tau(1-\tau)}}-1\right] 2^{\frac{R}{\tau}}
\end{aligned}
$$

The DMT of IDF is then given by

$$
d(r)=\min \left\{2-\frac{r}{1-\tau}, 2-\frac{r}{\tau}\right\}
$$

From (11), it is easy to see that when $\tau=1 / 2$, the curve of $d(r)$ is optimal, i.e., $d(r)=2-2 r$. Therefore, the DMT of IDF indeed achieves the MISO bound. 


\subsection{Selective Decode-and-Forward Protocol}

\subsubsection{Protocol Description}

In the first sub-slot, $\mathrm{S}$ broadcasts information to both the relay and $\mathrm{D}$. If the relay is unable to decode, $\mathrm{S}$ retransmits to $\mathrm{D}$ in the second sub-slot, or $\mathrm{S}$ keeps silent and the relay communicates with $\mathrm{D}$ in the second sub-slot.

\subsubsection{DMT Performance}

The maximum average mutual information can be readily shown as

$$
I_{S D F}=\left\{\begin{array}{c}
\log \left(1+\left|h_{s d}\right|^{2} S N R\right), \\
\text { when } \tau \log \left(1+\left|h_{s r}\right|^{2} S N R\right)<R \\
\tau \log \left(1+\left|h_{s d}\right|^{2} S N R\right)+(1-\tau) \log \left(1+\left|h_{r d}\right|^{2} S N R\right), \\
\text { when } \tau \log \left(1+\left|h_{s r}\right|^{2} S N R\right) \geq R
\end{array}\right.
$$

Let us first consider the following lemma [2].

Lemma : Let $\delta$ be a positive number and $r_{\delta}=$ $\delta f(v / \delta, w / \delta)$, where $v$ and $w$ are independent exponential random variables with parameters $\lambda_{v}$ and $\lambda_{w}$, respectively. Let $h(\delta)>0$ be continuous with $h(\delta) \rightarrow 0$ and $\delta / h(\delta) \rightarrow d<\infty$ as $\delta \rightarrow 0$. Then the probability $\operatorname{Pr}\left[r_{\delta}<\delta\right]$ satisfies

$$
\lim _{\delta \rightarrow 0} \frac{1}{h(\delta)} \operatorname{Pr}\left[r_{\delta}<h(\delta)\right]=\lambda_{v}+\lambda_{w}
$$

Armed with the above lemma, we then derive the outage probability of AF.

Let $U=1+\left|h_{s d}\right|^{2} S N R$, the probability density function of $U$ can be shown as

$$
P_{U}(u)=\frac{1}{\sigma_{s d}^{2} S N R} \exp \left(-\frac{1}{\sigma_{s d}^{2}} \frac{u-1}{S N R}\right)
$$

Letting $r_{\delta}=\frac{\left|h_{s r}\right|^{2}\left|h_{r d}\right|^{2}}{1+\left|h_{s r}\right|^{2}+\left|h_{r d}\right|^{2}}$, we have

The outage probability of SDF is derived as follows

$$
\begin{aligned}
& \left.P_{\text {out }}^{S D F}=\operatorname{Pr}\left[\tau \log \left(1+\left|h_{\text {sr }}\right|^{2} S N R\right)<R\right] \cdot \operatorname{Pr}\left[\log \left(1+\left|h_{\text {sd }}\right|^{2} S N R\right)<R\right]\right]^{A \text { out }}(R)=\operatorname{Pr}\left[(2 \tau-1) \log \left(1+\left|h_{\text {sd }}\right|^{2} S N R\right)+\right. \\
& +\operatorname{Pr}\left[\tau \log \left(1+\left|h_{s r}\right|^{2} S N R\right) \geq R\right] . \\
& \operatorname{Pr}\left[\tau \log \left(1+\left|h_{s d}\right|^{2} S N R\right)+(1-\tau) \log \left(1+\left|h_{r d}\right|^{2} S N R\right)<R\right] \\
& \left.(1-\tau) \log \left(1+\left|h_{s d}\right|^{2} S N R+\frac{\left|h_{s r}\right|^{2}\left|h_{r d}\right|^{2} S N R}{1+\left|h_{s r}\right|^{2}+\left|h_{r d}\right|^{2}}\right)<R\right] \\
& =\operatorname{Pr}\left[U^{2 \tau-1}\left(U+S N R \cdot r_{\delta}\right)^{1-\tau}<2^{R}\right]
\end{aligned}
$$

From (9), we have

$$
\begin{aligned}
P_{\text {out }}^{S D F} \sim & \frac{1}{\sigma_{s r}^{2} \sigma_{s d}^{2}}\left(\frac{2^{\frac{R}{\tau}}-1}{S N R}\right)\left(\frac{2^{R}-1}{S N R}\right)+ \\
& \frac{\lambda_{U} \lambda_{V}}{S N R^{2}} \frac{\tau}{2 \tau-1}\left[\left(2^{\frac{R}{1-\tau}}\right)^{\frac{2 \tau-1}{\tau}}-1\right] 2^{\frac{R}{\tau}}
\end{aligned}
$$

Thus, the DMT of SDF is

$$
d(r)=\min \left\{2-r-\frac{r}{\tau}, \min \left\{2-\frac{r}{1-\tau}, 2-\frac{r}{\tau}\right\}\right\}
$$

\section{DMT of AF Protocols}

\subsection{Amplify-and-Forward Protocol (AF)}

The maximum average mutual information is shown as

$$
\begin{aligned}
I_{A F}= & (2 \tau-1) \log \left(1+\left|h_{s d}\right|^{2} S N R\right)+ \\
& (1-\tau) \log \left(1+\left|h_{s d}\right|^{2} S N R+\frac{\left|h_{s r}\right|^{2}\left|h_{r d}\right|^{2} S N R}{1+\left|h_{s r}\right|^{2}+\left|h_{r d}\right|^{2}}\right)
\end{aligned}
$$

Based upon the above lemma and (18), we arrive at

$$
\begin{gathered}
P_{\text {out }}^{A F}(R)=\int_{0}^{2^{R / \tau}} \operatorname{Pr}\left[r_{\delta}<\frac{1}{S N R}\left[\left(\frac{2^{R}}{U^{2 \tau-1}}\right)^{\frac{1}{1-\tau}}-U\right]\right] P_{U}(u) d u \\
=\int_{0}^{2^{R / \tau}} \operatorname{Pr}\left[r_{\delta}<\frac{1}{S N R}\left[\left(\frac{2^{R}}{U^{2 \tau-1}}\right)^{\frac{1}{1-\tau}}-U\right]\right] . \\
\frac{1}{\sigma_{s d}^{2} S N R} \exp \left(-\frac{1}{\sigma_{s d}^{2} S N R} \frac{u-1}{S N R}\right) d u
\end{gathered}
$$

On the one hand, (20) can be upper-bounded by

$$
\begin{aligned}
& \int_{0}^{2^{R / \tau}} \operatorname{Pr}\left[r_{\delta}<\frac{1}{S N R}\left[\left(\frac{2^{R}}{U^{2 \tau-1}}\right)^{\frac{1}{1-\tau}}-U\right]\right] \frac{1}{\sigma_{s d}^{2} S N R} d u \\
& =\int_{0}^{2^{R / \tau}}\left(\frac{1}{\sigma_{s r}^{2}}+\frac{1}{\sigma_{r d}^{2}}\right) \frac{1}{S N R}\left[\left(\frac{2^{R}}{U^{2 \tau-1}}\right)^{\frac{1}{1-\tau}}-U\right] \frac{1}{\sigma_{s d}^{2} S N R} d u \\
& =\frac{2^{\frac{2 R}{\tau}}}{\sigma_{s d}^{2} S N R^{2}}\left(\frac{1}{\sigma_{s r}^{2}}+\frac{1}{\sigma_{r d}^{2}}\right)\left(\frac{1-\tau}{2-3 \tau}-\frac{1}{2}\right)
\end{aligned}
$$


On the other hand, (20) is lower-bounded by

$$
\begin{aligned}
& \int_{0}^{2^{R / \tau}} \operatorname{Pr}\left[r_{\delta}<\frac{1}{S N R}\left[\left(\frac{2^{R}}{U^{2 \tau-1}}\right)^{\frac{1}{1-\tau}}-U\right]\right] . \\
& \quad \frac{1}{\sigma_{s d}^{2} S N R}\left(1-\frac{1}{\sigma_{s d}^{2}} \frac{u-1}{S N R}\right) d u \\
&=\int_{0}^{2^{R / \tau}}\left(\frac{1}{\sigma_{s r}^{2}}+\frac{1}{\sigma_{r d}^{2}}\right) \frac{1}{S N R}\left[\left(\frac{2^{R}}{U^{2 \tau-1}}\right)^{\frac{1}{1-\tau}}-U\right] \frac{\left(1-\frac{1}{\sigma_{s d}^{2}} \frac{u-1}{S N R}\right)}{\sigma_{s d}^{2} S N R} d u \\
& \geq\left.\frac{1}{\sigma_{s r}^{2}}+\frac{1}{\sigma_{r d}^{2}}\right)\left(1-\frac{1}{\sigma_{s d}^{2}} \frac{2^{R / \tau}-1}{S N R}\right) \frac{1}{\sigma_{s d}^{2} S N R^{2}} . \\
& \int_{0}^{2^{R / \tau}}\left[\left(\frac{2^{R}}{U^{2 \tau-1}}\right)^{\frac{1}{1-\tau}}-U\right] d u \\
&=\left.\frac{1}{\sigma_{s r}^{2}}+\frac{1}{\sigma_{r d}^{2}}\right)\left(1-\frac{1}{\sigma_{s d}^{2}} \frac{2^{R / \tau}-1}{S N R}\right) \frac{2^{2 \frac{2 R}{\tau}}}{\sigma_{s d}^{2} S N R^{2}}\left(\frac{1-\tau}{2-3 \tau}-\frac{1}{2}\right)
\end{aligned}
$$

i.e. ,

$\liminf _{S N R \rightarrow \infty} P_{g\left(\frac{1}{S N R}\right) \rightarrow 0}^{A F} \geq \frac{2^{\frac{2 R}{\tau}}}{\sigma_{s d}^{2} S N R^{2}}\left(\frac{1}{\sigma_{s r}^{2}}+\frac{1}{\sigma_{r d}^{2}}\right)\left(\frac{1-\tau}{2-3 \tau}-\frac{1}{2}\right)$

As the upper bound equals the lower bound, we have

$$
P_{\text {out }}^{A F} \sim \frac{2^{\frac{2 R}{\tau}}}{\sigma_{s d^{2}}^{2} S N R^{2}}\left(\frac{1}{\sigma_{s r}^{2}}+\frac{1}{\sigma_{r d}^{2}}\right)\left(\frac{1-\tau}{2-3 \tau}-\frac{1}{2}\right)
$$

From (24), the DMT of AF can be seen as

$$
d(r)=2-\frac{2 r}{\tau}
$$

\subsection{Selective Amplify-and-Forward Protocol}

\subsubsection{Protocol Description}

In the first sub-slot, $\mathrm{S}$ broadcasts information to both the relay and $D$. If the relay failed the reception, $S$ repeats partial information to $\mathrm{D}$ in the second sub-slot, or $\mathrm{S}$ keeps silent and the relay amplifies and forwards.

\subsubsection{DMT Performance}

The maximum average mutual information can be readily shown as

$$
I_{S A F}=\left\{\begin{array}{c}
\log \left(1+\left|h_{s d}\right|^{2} S N R\right), \text { when } \tau \log \left(1+\left|h_{s r}\right|^{2} S N R\right)<R \\
(2 \tau-1) \log \left(1+\left|h_{s d}\right|^{2} S N R\right)+ \\
(1-\tau) \log \left(1+\left|h_{s d}\right|^{2} S N R+\frac{\left|h_{s r}\right|^{2}\left|h_{r d}\right|^{2} S N R}{1+\left|h_{s r}\right|^{2}+\left|h_{r d}\right|^{2}}\right), \\
\text { when } \tau \log \left(1+\left|h_{s r}\right|^{2} S N R\right) \geq R
\end{array}\right.
$$

From (26) and (24), the outage probability of SAF can be derived as follows

$$
\begin{aligned}
& P_{\text {out }}^{S A F}(R)= \\
& \operatorname{Pr}\left[\tau \log \left(1+\left|h_{s r}\right|^{2} S N R\right)<R\right] \operatorname{Pr}\left[\log \left(1+\left|h_{s d}\right|^{2} S N R\right)<R\right]+ \\
& \operatorname{Pr}\left[\tau \log \left(1+\left|h_{s r}\right|^{2} S N R\right) \geq R\right] \cdot \operatorname{Pr}\left[(2 \tau-1) \log \left(1+\left|h_{s d}\right|^{2} S N R\right)+\right. \\
& \left.(1-\tau) \log \left(1+\left|h_{s d}\right|^{2} S N R+\frac{\left|h_{s r}\right|^{2}\left|h_{r d}\right|^{2} S N R}{1+\left|h_{s r}\right|^{2}+\left|h_{r d}\right|^{2}}\right)<R\right] \\
& \sim \frac{1}{\sigma_{s r}^{2} \sigma_{s d}^{2}}\left(\frac{2^{\frac{R}{\tau}}-1}{S N R}\right)\left(\frac{2^{R}-1}{S N R}\right)+ \\
& \quad\left(\frac{1}{\sigma_{s r}^{2}}+\frac{1}{\sigma_{r d}^{2}}\right) \frac{2^{\frac{2 R}{\tau}}}{\sigma_{s d^{2}}^{2} S N R^{2}}\left(\frac{1-\tau}{2-3 \tau}-\frac{1}{2}\right)
\end{aligned}
$$

The DMT of SAF is calculated as follows

$$
d(r)=\min \left\{2-r-\frac{r}{\tau}, 2-\frac{2 r}{\tau}\right\}=2-\frac{2 r}{\tau}
$$

\subsection{Incremental Amplify-and-Forward Protocol}

\subsubsection{Protocol Description}

S transmits its information to D with spectral efficiency $\mathrm{R}$. Then D indicates the success or failure of the reception by broadcasting a single bit to feed back to $S$ and the relay. If successful, the relay keeps silent and $S$ continues to transmit new information to D. Otherwise, the relay forwards what it received from $\mathrm{S}$ and $\mathrm{S}$ does nothing.

\subsubsection{DMT Performance}

The maximum average mutual information can be readily shown to be

$$
I_{I A F}=\left\{\begin{array}{c}
\tau \log \left(1+\left|h_{s d}\right|^{2} S N R\right), \text { when } \tau \log \left(1+\left|h_{s d}\right|^{2} S N R\right) \geq R \\
(2 \tau-1) \log \left(1+\left|h_{s d}\right|^{2} S N R\right)+ \\
(1-\tau) \log \left(1+\left|h_{s d}\right|^{2} S N R+\frac{\left|h_{s r}\right|^{2}\left|h_{r d}\right|^{2} S N R}{1+\left|h_{s r}\right|^{2}+\left|h_{r d}\right|^{2}}\right), \\
\text { when } \tau \log \left(1+\left|h_{s d}\right|^{2} S N R\right)<R
\end{array}\right.
$$


From (29) and (24), the outage probability of IAF is derived as

$$
\begin{aligned}
& P_{\text {out }}^{I A F}(R)=\operatorname{Pr}\left[\tau \log \left(1+\left|h_{s d}\right|^{2} S N R\right)<R\right] . \\
& \operatorname{Pr}\left[(2 \tau-1) \log \left(1+\left|h_{s d}\right|^{2} S N R\right)+\right. \\
& (1-\tau) \log \left(1+\left|h_{s d}\right|^{2} S N R+\frac{\left|h_{s r}\right|^{2}\left|h_{r d}\right|^{2} S N R}{1+\left|h_{s r}\right|^{2}+\left|h_{r d}\right|^{2}}\right)< \\
& \left.\quad R \mid \tau \log \left(1+\left|h_{s d}\right|^{2} S N R\right)<R\right] \\
& =\operatorname{Pr}\left[(2 \tau-1) \log \left(1+\left|h_{s d}\right|^{2} S N R\right)+\right. \\
& \left.(1-\tau) \log \left(1+\left|h_{s d}\right|^{2} S N R+\frac{\left|h_{s r}\right|^{2}\left|h_{r d}\right|^{2} S N R}{1+\left|h_{s r}\right|^{2}+\left|h_{r d}\right|^{2}}\right)<R\right] \\
& \sim \frac{2^{\frac{2 R}{\tau}}}{\sigma_{s d}^{2} S N R^{2}}\left(\frac{1}{\sigma_{s r}^{2}}+\frac{1}{\sigma_{r d}^{2}}\right)\left(1-\frac{1}{\sigma_{s d}^{2}} \frac{2^{R / \tau}-1}{S N R}\right)\left(\frac{1-\tau}{2-3 \tau}-\frac{1}{2}\right)
\end{aligned}
$$

From (30), the DMT of IAF is obtained as

$$
d(r)=\min \left\{2-r-\frac{r}{\tau}, 2-\frac{2 r}{\tau}\right\}=2-\frac{2 r}{\tau}
$$

\section{Numerical Results}

To investigate the DMT performance of different relay protocols, numerical results are presented in this section.

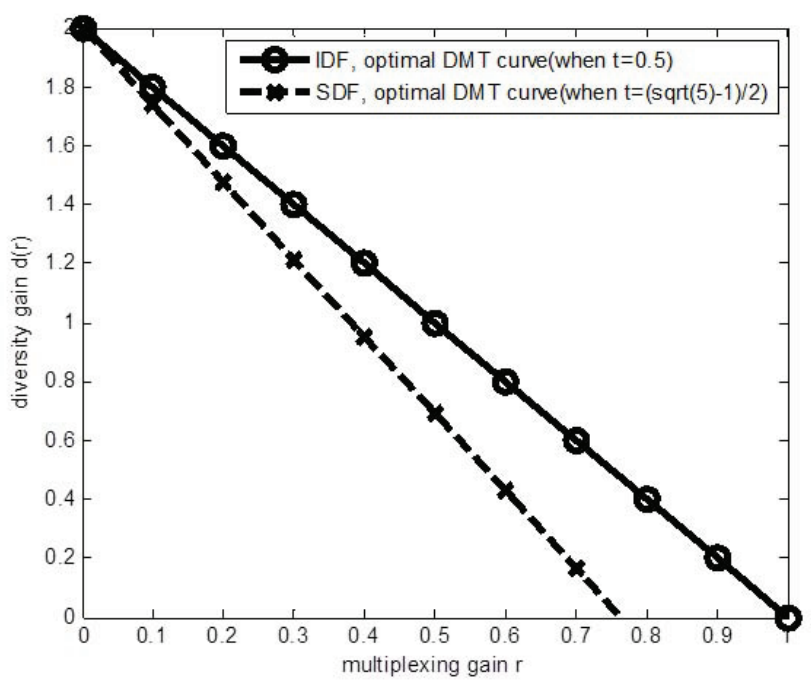

Fig. 2 Optimal DMT of IDF and SDF

Figure 2 shows the optimal DMT of IDF and SDF, from which we can see that any type of DF protocols can achieve its optimal DMT when the fraction of time $\tau$ for BS transmission is set properly. Among all the DF protocols, IDF with $\tau=0.5$ is the best, which can achieve the MISO upper bound irrespective of the multiplexing gain r. Furthermore, SDF obtains its optimal DMT when $\tau=(\sqrt{5}-1) / 2$.

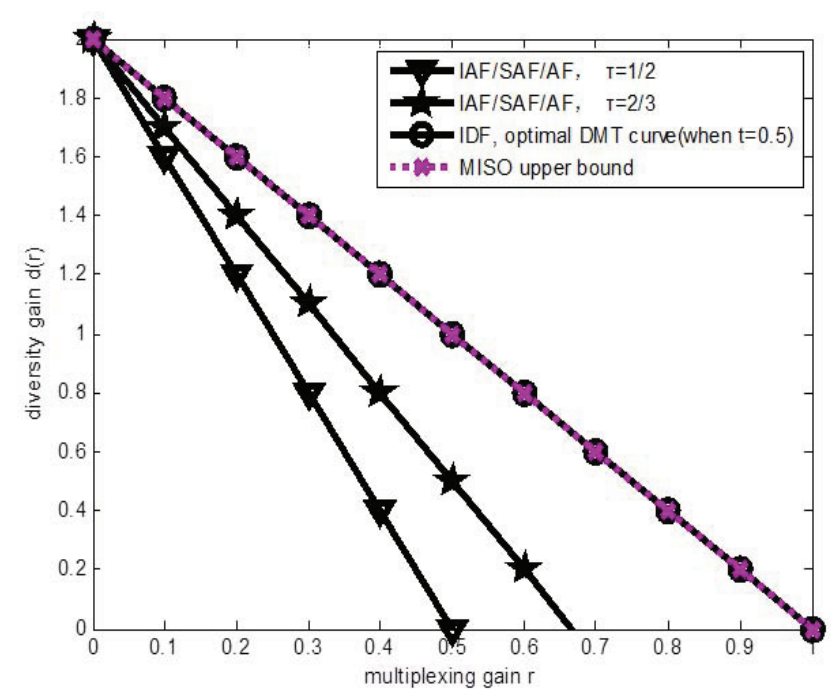

Fig. 3 Comparisons between the IDF and AF protocols.

Figure 3 shows that the DMT performance of the AF, SAF and IAF protocols is identical with any $\tau$. In contrast to the DF protocols, all the AF protocols have no optimal DMT curve in the entire range of multiplexing gain $r \in$ $(0,1)$. Moreover, when $\tau \rightarrow 1$, the maximal multiplexing gain of those AF protocols gets close to 1 and their DMT performance approaches the MISO upper bound.

\section{Conclusions}

In this paper, we classify various $\mathrm{AF}$ and $\mathrm{DF}$ relay protocols in terms of the DMT under the assumption that the transmitter only has the knowledge of the CDI. The DF protocols can be proven to achieve their optimal DMT when the lengths of the two sub-slots are in proper ratio. IDF is demonstrated to be superior to the other protocols when the two sub-slots are of equal length. IDF is shown to achieve the MISO upper bound for the entire range of the multiplexing gains. Moreover, SDF obtains its optimal DMT when the ratio is $\tau=(\sqrt{5}+1) / 2$. In addition, the DMT performance of the AF, SAF and IAF protocols is identical. They have no optimal DMT curve for $r \in(0,1)$. When the length of the first sub-slot tends to 1 , which indicates $\mathrm{S}$ transmits almost all the time while the relay works little time, the maximal multiplexing gain of these 
AF protocols increases up to 1 and the DMT performance approaches the MISO upper bound.

\section{Acknowledgement}

This paper is supported by Key project (2012ZX03001030-004).

\section{References}

[1] L. Zheng and D.N.C. Tse, "Diversity and multiplexing: A fundamental tradeoff in multiple-antenna channels", IEEE Trans. Inf. Theory, vol.49, no.5, pp. 1073-1096, May.2003.

[2] J. N. Laneman, D.N.C. Tse, and G. W. Wornell, "Cooperative diversity in wireless networks: Efficient protocols and outage behavior", IEEE Trans. Inf. Theory, vol. 50, no.12, pp.3062-3080, Dec.2004.

[3] R. U. Nabar, H. Bolcskei, and F. W. Kneubhler, "Fading relay channels:Performance limits and space-time signal design”, IEEE J. Select.Areas Commun., vol. 22, pp. 10991109, Aug. 2004.

[4] K. Azarian, H. E. Gamal, and P. Schniter, "On the achievable diversity-multiplexing tradeoff in half-duplex cooperative channels", IEEE Trans. Inf. Theory, vol. 51, pp. 4152-4172, Dec 2005.

[5] Sheng Yang and Jean-Claude Belfiore, "Towards the optimal amplify-and-forward cooperative diversity scheme", IEEE Trans. Inf. Theory, vol. 53, pp. 3114-3126, Sept. 2007.

[6] Sheng Yang and J.C.Belfiore, "On slotted amplify-andforward cooperative diversity schemes", Inf. Theory, IEEE.International Symposium, pp. 2446-2450, July.2006.

[7] M. Yuksel and E. Erkip, "Multiple-antenna cooperative wireless systems: diversity- multiplexing tradeoff perspective", IEEE Trans. Inf. Theory, vol. 53, no. 10, pp. 3371-3393, Oct. 2007.

[8] M.Yuksel and E.Erkip, ”Diversity-Multiplexing Tradeoff in Multiple-Antenna Relay System", Inf. Theory, IEEE.International Symposium, pp. 1154-1158, July.2006.

[9] M.Yuksel and E.Erkip, "Diversity-multiplexing Tradeoff in Half-Duplex Relay Systems”, ICC, pp.689-694, June 2007.

[10] D.N.C Tse and P.Viswanath, "Fundamentals of wireless communication", Cambridge University Press, 2005.

[11] T.M.Cover and A.A.El Gamal, "Capacity theorems for the relay channel", IEEE Trans. Inf. Theory, vol.25, no.5, pp. 572-584, Sep.1979.

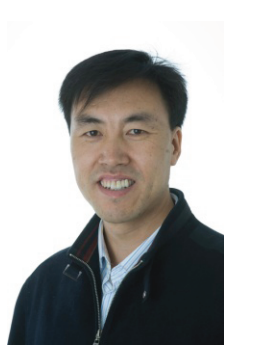

\section{Yafeng}

Wang

Shannxi

(S'00-M'03-SM'09)

China. Birthdate: November, 1974.

$\mathrm{He}$ received his $\mathrm{PhD}$ and MEng. in 2003 and 2000 respectively from the Beijing University of Posts and Telecommunications, the University of Electronic Science and Technology of China. And his research mainly focuses on wireless communications and information theory.In 2008, he worked as a visiting scholar in the Faculty of Engineering and Surveying at the University of Southern Queensland, Australia. He is an associate professor of electronic engineering in the School of Information and Telecommunications at the Beijing University of Posts and Telecommunications.

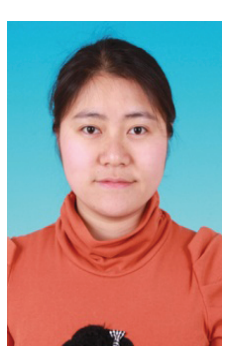

Lin Qin received her B.E. degree in communication engineering in Beijing University of Posts and Telecommunications. She has been a postgraduate student and studied in Wireless Theory \& Technology Lab in Beijing University of Posts and Telecommunications for a M.E. degree. Now she focuses on the research regarding innovative communication technologies in LTE-Advanced system.

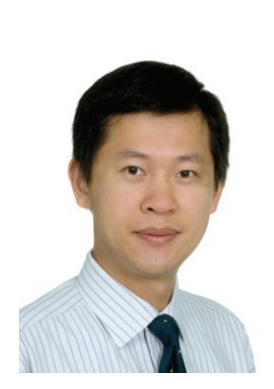

Wei Xiang (S'00-M'04-SM'10) received the B.Eng. and M.Eng. degrees, both in electronic engineering, from the University of Electronic Science and Technology of China, Chengdu, China, in 1997 and 2000, respectively, and the Ph.D. degree in telecommunications engineering from the University of South Australia, Adelaide, Australia, in 2004. Since January 2004, he has been with the Faculty of Engineering and Surveying, University of Southern Queensland, Toowoomba, Australia. In 2008, he was a visiting scholar at the School of Electrical and Electronic Engineering, Nanyang Technological University, Singapore. During Oct. 2010 and Mar. 2011, he was a visiting scholar at the University of Mississippi, Oxford, MS, USA. His research interests are in the broad area of communications and information theory, particularly coding and signal processing for multimedia communications systems. 